\title{
Efecto del volumen de empaque sobre la tasa de congelación-descongelación y la fertilidad de semen crioconservado de yamú (Brycon amazonicus)
}

\author{
Effects of packaged volume on freezing and thawing rates and the fertility \\ of cryopreserved sperm of yamú (Brycon amazonicus)
}

\author{
V M Medina-Robles, Y M Velasco-Santamaría, P E Cruz-Casallas* \\ Instituto de Acuicultura, Universidad de los Llanos, Villavicencio, Meta, Colombia
}

\section{SUMMARY}

\begin{abstract}
The aim of this study was to evaluate the freezing-thawing conditions of yamú (Brycon amazonicus) sperm packaged in straws of different volume and their effect on the spermatic motility and fertility post-thawing. The induction to gonadal final maturation was made with carp pituitary extract. The sperm obtained was evaluated and diluted (1:4) in a solution with $5.5 \%$ glucose, $12 \%$ egg yolk and $10 \%$ dimethyl sulfoxide, packaged in $0.5,1.8,2.5$ or $4.0 \mathrm{ml}$ straws and frozen in nitrogen vapors. The thawing was carried out in a $35^{\circ} \mathrm{C}, 60^{\circ} \mathrm{C}$ or $80^{\circ} \mathrm{C}$ water bath during different times. All straws showed a similar overall freezing rate $\left(7.5\right.$ to $\left.12.9^{\circ} \mathrm{C} \mathrm{min}{ }^{-1}\right)$. On the other hand, the overall thawing rate was faster at a higher water bath temperature. The $4.0 \mathrm{ml}$ straws thawed at $35^{\circ} \mathrm{C}$ showed the highest motility and activation time post-thaw $(47.0 \pm 1.6 \%$ and $60.2 \pm 2.4 \mathrm{sec})$. The fertility obtained with cryopreserved sperm in all straw sizes was significantly lower $(\mathrm{P}<0.05)$ than with fresh sperm $(65 \pm 4 \%)$. The highest fertility was observed with $0.5 \mathrm{ml}$ straw thawed at $35^{\circ} \mathrm{C}(52 \pm 3 \%)$, followed by $2.5,4.0$ and $1.8 \mathrm{ml}$ straws $(51 \pm 3,48 \pm 3$ and $47 \pm 3 \%$, respectively). It was concluded that $4.0 \mathrm{ml}$ straws thawed at $35^{\circ} \mathrm{C}$ showed the best post-thaw sperm quality and more homogenous freezing-thawing rates in comparison to the other packaging systems. On the other hand, no effects on fertility were observed in all straw sizes thawed at $35^{\circ} \mathrm{C}$.
\end{abstract}

Palabras clave: fertilidad, pajilla, semen, tasa de congelación-descongelación.

Key words: fertility, freezing-thawing rate, sperm, straw.

\section{INTRODUCCION}

La crioconservación es el método más utilizado actualmente para el almacenamiento de semen de peces por períodos prolongados de tiempo. Sin embargo, aún muchas investigaciones son orientadas a la determinación de protocolos más eficientes, estudiando diversas mezclas de soluciones crioprotectoras y evaluando diferentes tasas, tanto de descenso de la temperatura como de descongelación del material seminal (Medina-Robles y col 2005). Pocos autores realizan una descripción detallada de las curvas de congelación y descongelación utilizadas en sus ensayos (Cabrita y col 2001, Cruz-Casallas y col 2006), no siendo posible en todos los casos analizar la eventual asincronía entre las velocidades de congelación y descongelación, la cual es responsable de la disminución de la calidad espermática del semen descongelado debido al criodaño sobre la célula espermática.

Los sistemas de empacado utilizados actualmente incluyen envases de diferentes volúmenes, que van desde microtubos de 0,08 $\mathrm{ml}$ hasta macrotubos de 5,0 ml. También son empleados pellets $(0,07-0,1 \mathrm{ml})$ y pajillas plásticas de pequeño volumen $(0,25-0,5 \mathrm{ml})$, siendo es-

Aceptado: 16.01.2007.

* pecruz@telecom.com.co tas últimas utilizadas con éxito en varias especies de peces (Lahnsteiner y col 1996, Lahnsteiner 2000, CruzCasallas y col 2004). En el proceso de crioconservación usualmente se producen daños en un gran número de espermatozoides, siendo sólo una pequeña fracción de células espermáticas viable después de la descongelación del semen (Ogier de Baulny y col 1997), lo cual determina que para el proceso de seminación artificial con semen crioconservado, bajo las condiciones de granjas productoras de alevinos, se requiera utilizar grandes volúmenes (Billard 1998, Cruz-Casallas y col 2006). La cantidad de semen empacado en pajillas de $0,5 \mathrm{ml}$ es insuficiente para fertilizar desoves de especies de alta fecundidad. Por lo tanto, el empleo de macrotubos que permiten congelar volúmenes mayores de semen facilita los procesos de descongelación y de seminación artificial a escala comercial (Lahnsteiner 2000). En salmónidos se ha evaluado el uso de macrotubos para la congelación seminal y para su utilización en granjas, mostrando aún resultados variables (Lahnsteiner y col 1997, Cabrita y col 2001), lo cual amerita en estos sistemas de empaque evaluar su utilización y comportamiento durante el proceso de congelación-descongelación en cada especie.

El yamú (Brycon amazonicus) es un carácido endémico de la Orinoquía Colombiana, con alto potencial productivo que lo ubica dentro de las principales especies ícticas para la acuicultura nacional. Sin embargo, 
debido a su asincronismo en la maduración gonadal y a su alta fecundidad, la implementación de la crioconservación seminal en empaques de grandes volúmenes podría optimizar los procesos de seminación artificial en esta especie. A nivel de laboratorio se han realizado varios experimentos (Cruz-Casallas y col 2004) con resultados satisfactorios, similares a aquellos obtenidos en otras especies ícticas cultivadas, permitiendo actualmente su aplicación a escala comercial; no obstante, el perfeccionamiento de los procesos de congelación y descongelación contribuirá a mejorar las tasas de fertilidad observadas hasta ahora.

En consecuencia, el objetivo de este estudio fue establecer las condiciones de congelación-descongelación para semen de yamú en diferentes volúmenes de empaque y relacionarlas con algunos parámetros de calidad seminal postdescongelación, tales como la movilidad espermática y la fertilidad.

\section{MATERIAL Y METODOS}

Animales. Fueron utilizados hembras y machos adultos de yamú (Brycon amazonicus) nacidos y criados en cautiverio, provenientes del grupo de reproductores de la Estación Piscícola de la Universidad de los Llanos. Los reproductores fueron mantenidos en estanques de tierra a una densidad de $200 \mathrm{~g} / \mathrm{m}^{2}$, con recambio de agua diario de aproximadamente el 5\%. La alimentación se realizó una vez por día, seis veces por semana, a razón del $3 \%$ del peso vivo, con alimento peletizado de $25 \%$ de proteína bruta.

La selección de los ejemplares se efectuó con base en su desarrollo corporal y en su condición de madurez sexual. En el caso de los machos, esta última se evaluó por la presencia de semen en la papila urogenital después de un leve masaje craneocaudal del abdomen, mientras que en las hembras se determinó mediante biopsia ovárica y evaluación estereoscópica del estado de desarrollo de los oocitos. Los experimentos fueron realizados durante la época reproductiva (marzo a mayo) de 2005.

Inducción a la maduración final de las gónadas. La maduración final y ovulación fueron realizadas mediante la aplicación de una dosis total de $5,75 \mathrm{mg} / \mathrm{kg}$ de peso corporal de extracto de hipófisis de carpa (EHC, Stoller Fisheries, USA), distribuida en tres aplicaciones: 0,25, 0,50 y 5,00 $\mathrm{mg} / \mathrm{kg}$, separadas por 24 y $12 \mathrm{~h}$, respectivamente. La espermiación fue llevada a cabo por la administración de una única inyección intramuscular de $\mathrm{EHC}$, equivalente a 4 $\mathrm{mg} / \mathrm{kg}$ de peso corporal, aplicada simultáneamente con la segunda dosis de inducción de la hembra.

Obtención de gametos. Previo a la obtención de los gametos, los animales fueron tranquilizados por inmersión en una solución de 300 ppm de 2-fenoxietanol (Sigma Co., St. Louis, Missouri) durante 3 a 5 min. En las hembras la ovulación ocurrió aproximadamente $6 \mathrm{~h}$ después de la última dosis de EHC e inmediatamente después los oocitos fueron recolectados en recipientes plásticos secos aforados. El semen fue colectado $18 \mathrm{~h}$ después de la aplicación de la hormona en tubos de vidrio graduados estériles. En ambos casos fue realizado un leve masaje en sentido craneocaudal de la región abdominal para la obtención de los gametos, evitando contaminación con agua, heces, bilis u orina.

Evaluación seminal. Una vez colectada la muestra de semen fueron registrados el volumen y el color. La movilidad fue estimada subjetivamente bajo microscopio óptico (100X), después de la activación de una gota de semen $(20 \mu \mathrm{l})$ con agua destilada o bicarbonato de sodio $\left(\mathrm{NaHCO}_{3}\right)$ al $1 \%(180 \mu \mathrm{l})$ para semen fresco y semen descongelado, respectivamente, y expresada en porcentaje. El tiempo de activación fue medido desde la inducción de la movilidad espermática hasta la suspensión de movimientos y expresado en segundos. La concentración espermática fue determinada por conteo en cámara de Neubauer, previa dilución del semen 1:1200 con solución salina fisiológica $(0,9 \% \mathrm{NaCl})$. Todas las evaluaciones fueron realizadas dentro de los $30 \mathrm{~min}$ siguientes a la colección del semen. Muestras seminales con movilidades inferiores a $80 \%$ no fueron procesadas.

Congelación del semen. Muestras seminales de igual volumen fueron mezcladas para constituir un pool de semen de cuatro machos. El material seminal fue diluido en proporción 1:4 (semen:diluyente), en una solución constituida por $5,5 \%$ de glucosa (p:v), $12 \%$ de yema de huevo (v:v) y $10 \%$ (v:v) de dimetil sulfóxido (DMSO, Sigma Chemical Co., St. Louis, MO, USA). Inmediatamente después fue empacado en pajillas de 0,5 ml (IMV, Instrument de Médecine Vétérinaire, Minneapolis, USA), 1,8, 2,5 o 4,0 ml (Minitub, Abfül-und Labortechnik $\mathrm{GmbH} \&$ Co. KG) y selladas con polivinilo y esferas metálicas. Las pajillas de $0,5 \mathrm{ml}$ fueron selladas únicamente con polivinilo. Para la congelación, las pajillas fueron distribuidas aleatoriamente en un soporte de polímero de PVC, diseñado para mantenerlas en posición vertical y lograr una congelación homogénea, el cual fue inmediatamente introducido en un termo de vapores (dry shipper) de nitrógeno (Taylor-Wharton, CP 100, Theodore, AL, USA) durante $30 \mathrm{~min}$. Finalmente, todas las pajillas fueron trasladadas a un termo de almacenamiento, sumergiéndolas directamente en nitrógeno líquido (NL) a $-196^{\circ} \mathrm{C}$ (Taylor-Wharton, HC 35, Theodore, AL, USA) hasta la realización de los experimentos. Este procedimiento fue repetido cinco veces, empleando machos diferentes en cada uno de ellos.

Evaluación de las condiciones de congelación y descongelación. Todas las pajillas fueron congeladas como se indicó anteriormente y descongeladas por inmersión en 
baños de agua a $35,60 \mathrm{u} 80^{\circ} \mathrm{C}$. La temperatura dentro de la pajilla fue monitoreada con una termocupla (WBrand, USA, precisión $0,01^{\circ} \mathrm{C}$ y rango de -200 a $800^{\circ} \mathrm{C}$ ) insertada directamente en el centro de la pajilla. Las mediciones de descenso de temperatura se realizaron a partir de la temperatura ambiente del laboratorio $\left(28^{\circ} \mathrm{C}\right)$ hasta alcanzar $-196^{\circ} \mathrm{C}$, así: cada 5 seg durante el primer minuto, cada 10 seg durante los tres minutos siguientes y cada 15 seg hasta $-196^{\circ} \mathrm{C}$. Durante el proceso de descongelación, el ascenso de la temperatura fue registrado cada $5 \mathrm{seg}$ desde $-196^{\circ} \mathrm{C}$ hasta alcanzar la temperatura del baño de agua de descongelación. La tasa de congelación fue analizada desde $28^{\circ} \mathrm{C} \mathrm{a}-20^{\circ} \mathrm{C}$, desde $-20^{\circ} \mathrm{C}$ hasta $-100^{\circ} \mathrm{C}$ y desde $-100^{\circ} \mathrm{C}$ hasta $-196^{\circ} \mathrm{C}$ y la tasa de descongelación fue analizada desde $-196^{\circ} \mathrm{C}$ hasta $0^{\circ} \mathrm{C}$ y desde $0^{\circ} \mathrm{C}$ hasta $35^{\circ} \mathrm{C}$. La tasa total de congelación y descongelación fue determinada desde $28^{\circ} \mathrm{C}$ hasta $-196^{\circ} \mathrm{C}$ y desde $-196^{\circ} \mathrm{C}$ hasta $35^{\circ} \mathrm{C}$, respectivamente. Todas las curvas de congelación-descongelación fueron realizadas por triplicado.

Evaluación de la calidad espermática postdescongelación. Para este experimento fue utilizado un arreglo factorial 4 x 3 (4 volúmenes de empaque y 3 temperaturas de descongelación) para un total de 12 tratamientos. Tres pajillas de cada volumen de empaque por pool congelado fueron descongeladas a las tres temperaturas descritas anteriormente y durante diferentes tiempos, como se muestra en el cuadro 1, para un total de 15 pajillas por tratamiento. Inmediatamente después de descongelado el semen, fueron determinados la movilidad espermática y el tiempo de activación, siguiendo el procedimiento descrito anteriormente.

Para los ensayos de fertilidad, $2 \mathrm{~g}$ de oocitos ( $c a .2 .800$ oocitos) fueron seminados con semen descongelado de cada uno de los tratamientos empleando dosis ajustadas a 75.000 espermatozoides móviles/oocito y activados con

Cuadro 1. Tiempo de descongelación (seg) de semen de yamú (Brycon amazonicus) empacado en pajillas de $0,5,1,8,2,5$ o $4,0 \mathrm{ml}$, sometido a tres diferentes temperaturas. La congelación fue realizada en vapores de nitrógeno durante 30 min y la descongelación en baño de agua.

Thawing time (sec) of yamú (Brycon amazonicus) sperm packaged in $0.5,1.8,2.5$ or $4.0 \mathrm{ml}$ straws, submitted to three different temperatures. The freezing was carried out in nitrogen vapors during $30 \mathrm{~min}$ and the thawing in water bath.

\begin{tabular}{|c|c|c|c|}
\hline \multirow{2}{*}{$\begin{array}{l}\text { Volumen de } \\
\text { la pajilla (ml) }\end{array}$} & \multicolumn{3}{|c|}{$\begin{array}{c}\text { Temperatura de } \\
\text { descongelación }\left({ }^{\circ} \mathrm{C}\right)\end{array}$} \\
\hline & 35 & 60 & 80 \\
\hline 0,5 & 90 & 15 & 10 \\
\hline 1,8 & 90 & 40 & 25 \\
\hline 2,5 & 90 & 40 & 25 \\
\hline 4,0 & 90 & 40 & 25 \\
\hline
\end{tabular}

$\mathrm{NaHCO}_{3}$ al 1\%, de acuerdo con lo reportado por VelascoSantamaría y col (2004). Posteriormente se permitió un período mínimo de 10 min para la hidratación de los oocitos, por medio de lavados sucesivos con agua proveniente de los tanques de incubación. Luego de hidratados, fueron ubicados en incubadoras experimentales de flujo ascendente de 1,5 1, con el fin de facilitar la continuación del proceso de embriogénesis. La fertilidad se determinó seis horas después de la activación espermática, a partir de tres muestras de aproximadamente 100 oocitos cada una, recolectadas al azar de cada incubadora, mediante un tubo de vidrio de $50 \mathrm{~cm}$ de longitud. El porcentaje de fertilidad se calculó con base en el número de oocitos embrionados (estado de cierre del blastoporo) sobre el total contenido en la muestra. Como control se evaluó la misma cantidad de oocitos, seminados con semen fresco utilizando una dosis de 50.000 espermatozoides móviles/ oocito (Velasco-Santamaría y col 2004). Para cada pool de semen evaluado fue utilizada una hembra diferente.

Análisis estadístico. Los datos obtenidos fueron organizados y sometidos a estadística descriptiva y expresados como media \pm error estándar (EE). Los valores de las variables estimadas en porcentaje fueron transformados en arcoseno. Posteriormente, todos los resultados fueron sometidos a análisis de varianza de dos vías (ANOVA), seguido de la prueba de comparación de Duncan cuando hubo diferencia significativa. Previo a cada análisis, la homogeneidad de las varianzas fue establecida por medio de la prueba de Bartlett. En todos los casos $\mathrm{P}<0,05$ reveló diferencia significativa. Todos los procedimientos estadísticos fueron realizados empleando el software SAS versión 8.02 para Windows (1999-2001 por SAS Institute Inc., Cary, NC, USA).

\section{RESULTADOS}

\section{ANALISIS DE LAS CONDICIONES \\ DE CONGELACION-DESCONGELACIÓN}

La tasa de congelación para las pajillas de 1,8, 2,5 y $4,0 \mathrm{ml}$, en los tres rangos establecidos, fue similar, siendo menor en la pajilla de 4,0 ml; sin embargo, este empaque presentó un descenso más homogéneo desde $28^{\circ} \mathrm{C}$ hasta $-100^{\circ} \mathrm{C}$ (cuadro 2). Todas las pajillas mostraron una tasa total de congelación similar, fluctuando entre 7,5 y $12,9^{\circ} \mathrm{C} \min ^{-1}$. Las pajillas de $0,5 \mathrm{ml}$ presentaron el descenso más rápido de temperatura desde $28^{\circ} \mathrm{C}$ hasta $-20^{\circ} \mathrm{C}$. En contraste, la mayor velocidad de congelación se registró desde $-20^{\circ} \mathrm{C}$ hasta $-100^{\circ} \mathrm{C}$ en los macrotubos (cuadro 2). La temperatura de $-100^{\circ} \mathrm{C}$ fue alcanzada más rápidamente por la pajilla de $0,5 \mathrm{ml}(1 \mathrm{~min})$, seguida por las pajillas de 1,8 y $2,5 \mathrm{ml}(3,5 \mathrm{~min})$ y finalmente por la pajilla de 4,0 $\mathrm{ml}$ (4,5 min) (figura 1). En todos los sistemas de empaque se presentó una tasa de congelación muy lenta desde $-100^{\circ} \mathrm{C}$ hasta $-196^{\circ} \mathrm{C}$, alcanzando esta últi- 
ma temperatura aproximadamente a los 19 min de congelación (cuadro 2, figura 1). Cerca de 1,5 min después de iniciado el proceso de congelación, todas las pajillas, a excepción de $0,5 \mathrm{ml}$, presentaron un evidente pico de ascenso de temperatura entre $0^{\circ} \mathrm{C} \mathrm{y}-20^{\circ} \mathrm{C}$ (figura 1).

Las pajillas de $0,5 \mathrm{ml}$ en general presentaron las mayores tasas totales de descongelación, cuando fueron comparadas con los otros volúmenes de empaque en las tres temperaturas de descongelación estudiadas, sien-

Cuadro 2. Tasa de congelación $\left({ }^{\circ} \mathrm{C} \min ^{-1}\right)$ de semen de yamú (Brycon amazonicus) expuesto a vapores de nitrógeno y crioconservado en pajillas de $0,5,1,8,2,5$ o 4,0 ml. Los datos corresponden a la media de tres réplicas para cada volumen de empaque.

Freezing rate $\left({ }^{\circ} \mathrm{C} \mathrm{min}^{-1}\right)$ of yamú (Brycon amazonicus) sperm exposed to nitrogen vapors and cryopreserved in $0.5,1.8,2.5$ or $4.0 \mathrm{ml}$ straws. The values correspond to a mean of three replicates for each packaged volume.

\begin{tabular}{ccccr}
\hline \multirow{2}{*}{$\begin{array}{c}\text { Volumen } \\
\text { de la } \\
\text { pajilla } \\
(\mathrm{ml})\end{array}$} & \multicolumn{4}{c}{$\begin{array}{c}\text { Rango de temperatura } \\
\text { de congelación }\left({ }^{\circ} \mathrm{C}\right)\end{array}$} \\
\cline { 2 - 5 } & $28 \mathrm{a}-20$ & $-20 \mathrm{a}-100$ & $-100 \mathrm{a}-196$ & Total \\
\hline 0,5 & 151,5 & 96,4 & 3,2 & 7,5 \\
1,8 & 30,1 & 43,5 & 6,3 & 7,5 \\
2,5 & 30,1 & 43,5 & 6,8 & 12,9 \\
4,0 & 27,3 & 29,9 & 5,5 & 10,6 \\
\hline
\end{tabular}

do las más altas cuando fueron descongeladas a $60^{\circ} \mathrm{C} \mathrm{y}$ $80^{\circ} \mathrm{C}$ (cuadro 3). En las tres temperaturas evaluadas, todas las pajillas presentaron la mayor tasa de descongelación desde $-196^{\circ} \mathrm{C}$ hasta $0^{\circ} \mathrm{C}$ (cuadro 3). Las pajillas de 2,5 y $4,0 \mathrm{ml}$, descongeladas a $60^{\circ} \mathrm{C}$ y $80^{\circ} \mathrm{C}$, presentaron tasas de descongelación iguales desde $-196^{\circ} \mathrm{C}$ hasta $0^{\circ} \mathrm{C}$ e inferiores a la observada en las pajillas de 1,8 $\mathrm{ml}$ (cuadro 3).

En general, para todos los volúmenes de empaque la tasa total de descongelación aumentó a medida que se utilizó una mayor temperatura de descongelación (cuadro 3). La temperatura del semen descongelado al momento de ser utilizado fue de $35,4 \pm 1,1^{\circ} \mathrm{C}$, la cual fue alcanzada, por todos los volúmenes de empaque a los $c a$. $90 \mathrm{seg}$, cuando se utilizó la temperatura de descongelación de $35^{\circ} \mathrm{C}$ (figura $2 \mathrm{~A}$ ); cuando se empleó temperatura de $60^{\circ} \mathrm{C}$, ésta fue alcanzada en $\mathrm{ca} .15 \mathrm{seg}$ por las pajillas de $0,5 \mathrm{ml} \mathrm{y} \mathrm{ca.} 40 \mathrm{seg}$ por los macrotubos (figura 2B). Finalmente, en el baño de agua a $80^{\circ} \mathrm{C}$, las pajillas de 0,5 $\mathrm{ml}$ alcanzaron $\operatorname{los} 35,4 \pm 1,1^{\circ} \mathrm{C}$ a los $c a$. $10 \mathrm{seg}$ y por los otros tres volúmenes de empaque a los ca. $25 \mathrm{seg}$ (figura 2C).

\section{CALIDAD ESPERMATICA POSTDESCONGELACION}

Movilidad y tiempo de activación espermática. Con relación a la movilidad espermática, sólo las pajillas de $0,5 \mathrm{ml}$ descongeladas a $60^{\circ} \mathrm{C} \mathrm{u} 80^{\circ} \mathrm{C}$ mostraron una caída drástica y altamente significativa $(\mathrm{P}<0,05)$, cuando

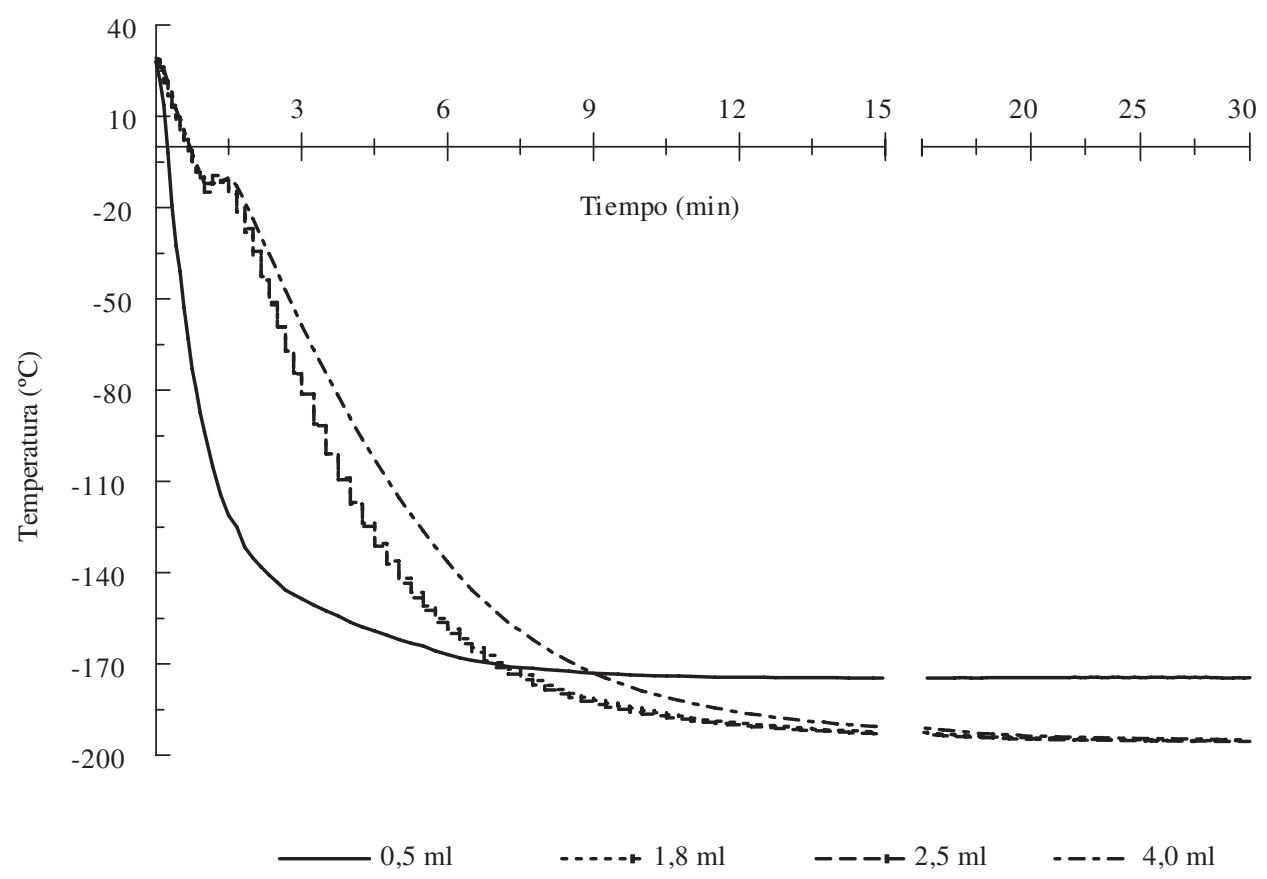

Figura 1. Curvas de congelación de semen de yamú (Brycon amazonicus) empacado en pajillas de $0,5,1,8,2,5$ o 4,0 $\mathrm{ml}$ y congelado en vapores de nitrógeno.

Freezing curves of yamú (Brycon amazonicus) sperm packaged in $0.5,1.8,2.5$ or $4.0 \mathrm{ml}$ straws and frozen in nitrogen vapors. 
Cuadro 3. Tasa de descongelación $\left({ }^{\circ} \mathrm{C} \min { }^{-1}\right)$ de semen de yamú (Brycon amazonicus) crioconservado en pajillas de 0,5 , $1,8,2,5$ o $4,0 \mathrm{ml}$ y descongelados en baño de agua a diferentes temperaturas. Los datos corresponden a la media de 3 réplicas de cada volumen de pajilla para cada temperatura de descongelación.

Thawing rate $\left({ }^{\circ} \mathrm{C} \min ^{-1}\right.$ ) of yamú (Brycon amazonicus) sperm cryopreserved in $0.5,1.8,2.5$ or $4.0 \mathrm{ml}$ straws and thawed in water bath at different temperatures. The values correspond to a mean of three replicates of each straw volume for each thawing temperature.

\begin{tabular}{ccrrr}
\hline \multirow{2}{*}{$\begin{array}{c}\text { Volumen } \\
\text { de la } \\
\text { pajilla }(\mathrm{ml})\end{array}$} & $\begin{array}{c}\text { Temperatura } \\
\text { descongelación } \\
\left({ }^{\circ} \mathrm{C}\right)\end{array}$ & \multicolumn{3}{c}{$\begin{array}{c}\text { Rango de temperatura de } \\
\text { descongelación }\left({ }^{\circ} \mathrm{C}\right)\end{array}$} \\
\cline { 3 - 5 } & & -196 a 0 & 0 a 35 & Total \\
\hline \multirow{2}{*}{0,5} & 35 & 824,0 & 152,9 & 552,4 \\
& 60 & $\mathrm{ND}$ & $\mathrm{ND}$ & $1.035,2$ \\
& 80 & $\mathrm{ND}$ & $\mathrm{ND}$ & $1.123,8$ \\
1,8 & 35 & 479,3 & 35,3 & 173,8 \\
& 60 & 587,9 & 102,1 & 344,6 \\
& 80 & 776,0 & 140,0 & 436,0 \\
2,5 & 35 & 336,0 & 23,9 & 106,4 \\
& 60 & 461,9 & 70,0 & 342,4 \\
& 80 & 587,8 & 205,8 & 444,0 \\
4,0 & 35 & 389,2 & 20,0 & 99,2 \\
& 60 & 461,9 & 140,0 & 340,0 \\
& 80 & 587,8 & 205,8 & 417,8 \\
\hline
\end{tabular}

ND: No determinado, debido al ascenso rápido de temperatura dentro de la pajilla.

fueron comparadas con las descongeladas a $35^{\circ} \mathrm{C}$. $\mathrm{Al}$ comparar el efecto de la temperatura sobre cada volumen de empaque, únicamente las pajillas de 0,5 y $4,0 \mathrm{ml}$ descongeladas a $60^{\circ} \mathrm{C}$ u $80^{\circ} \mathrm{C}$ presentaron menor $(\mathrm{P}<0,05)$ movilidad espermática que cuando fueron descongeladas a $35^{\circ} \mathrm{C}$. Por otro lado, las pajillas de $4,0 \mathrm{ml}$ descongeladas a $35^{\circ} \mathrm{C}$ presentaron diferencias significativas con las pajillas de 0,5 y $2,5 \mathrm{ml}$ (cuadro 4). En general, las pajillas de $0,5 \mathrm{ml}$ descongeladas a $60^{\circ} \mathrm{C}$ u $80^{\circ} \mathrm{C}$ mostraron los menores $(\mathrm{P}<0,05)$ porcentajes de movilidad. El tiempo de activación no mostró marcadas diferencias entre los volúmenes de empaque; sin embargo, el menor tiempo de activación fue registrado en las pajillas de $0,5 \mathrm{ml}$ descongeladas a $80^{\circ} \mathrm{C}$, el cual fue significativamente diferente $(\mathrm{P}<0,05)$ de los otros volúmenes de empaque. El mayor tiempo de activación espermática fue observado en las pajillas de $1,8 \mathrm{ml}$ descongeladas a $60^{\circ} \mathrm{C}$, siendo únicamente diferente $(\mathrm{P}<0,05)$ al del semen empacado en pajillas de $0,5 \mathrm{ml}$ descongeladas a $60^{\circ} \mathrm{C} \mathrm{u} 80^{\circ} \mathrm{C}$ (cuadro 4 ).

Ensayos de fertilidad. Los porcentajes de fertilidad obtenidos con semen congelado en todos los volúmenes de empaque y temperaturas de descongelación fueron significativamente menores $(\mathrm{P}<0,05)$ a aquellos observados con semen fresco $(64,4 \pm 3,9)$ (figura 3$)$. En todas las pajillas la fertilidad disminuyó a medida que la tem-

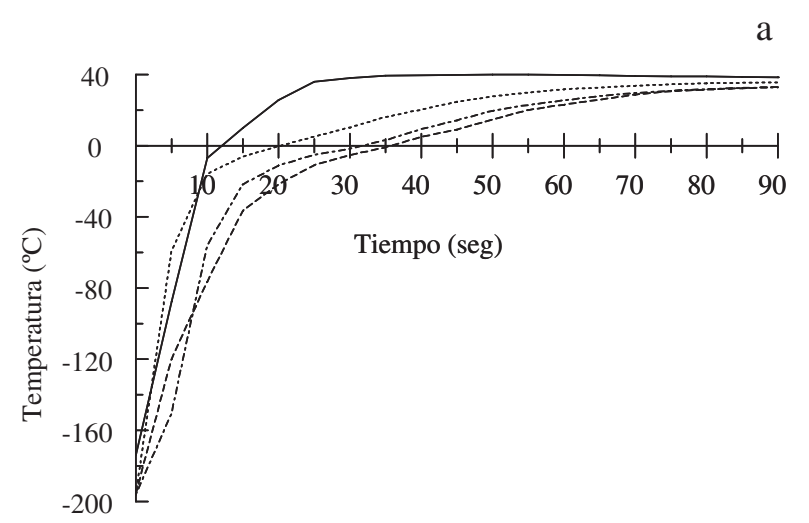

b
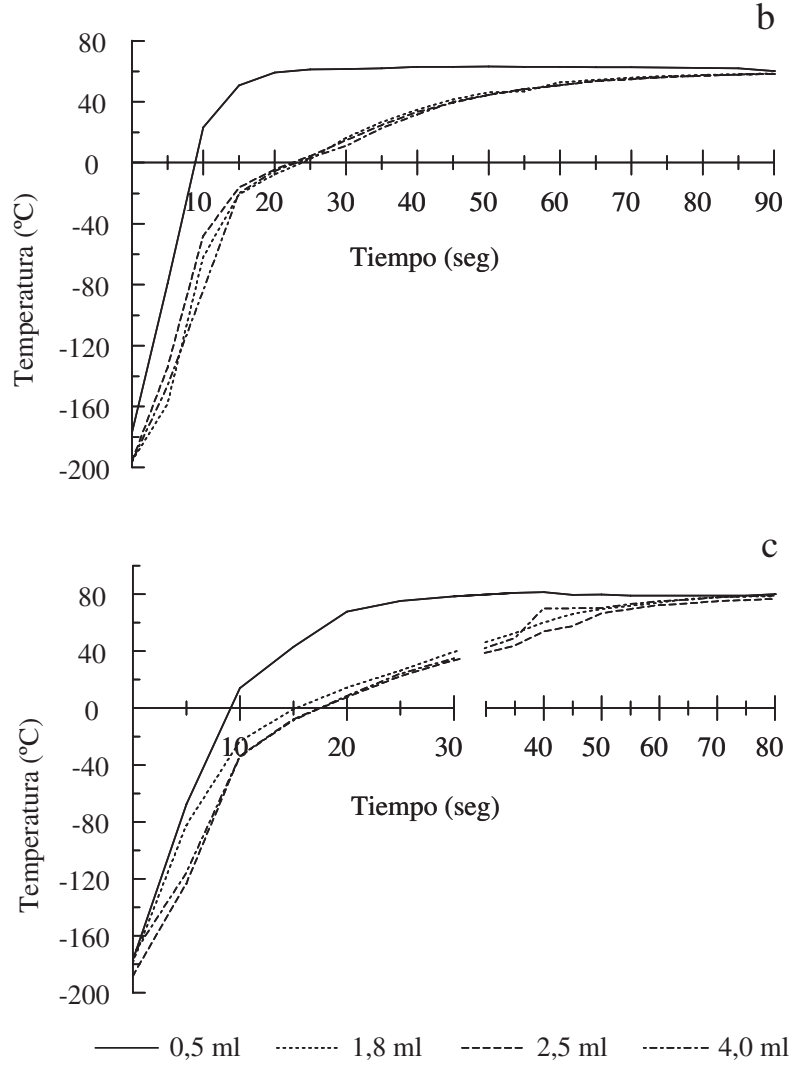

Figura 2. Curvas de descongelación de semen de yamú (Brycon amazonicus) empacado en pajillas de 0,5, 1,8, 2,5 o $4,0 \mathrm{ml}$ y descongelado a $35^{\circ} \mathrm{C}$ (a), $60^{\circ} \mathrm{C}$ (b) u $80^{\circ} \mathrm{C}$ (c). Los datos fueron tomados desde nitrógeno líquido hasta alcanzar la temperatura del baño de agua de descongelación.

Thawing curves of yamú (Brycon amazonicus) sperm packaged in $0.5,1.8,2.5$ or $4.0 \mathrm{ml}$ straws and thawed at $35^{\circ} \mathrm{C}$ (a), $60^{\circ} \mathrm{C}$ (b) or $80^{\circ} \mathrm{C}$ (c). The values were registered from liquid nitrogen up to reaching temperature of thawing water bath.

peratura de descongelación aumentó, a excepción de las pajillas de $0,5 \mathrm{ml}$, en las cuales la menor fertilidad fue registrada a $60^{\circ} \mathrm{C}(19,7 \pm 5,1 \%)$ (figura 3$)$. El más alto porcentaje de fertilidad fue mostrado por las pajillas de $0,5 \mathrm{ml}$ descongeladas a $35^{\circ} \mathrm{C}(51,6 \pm 3,4)$, seguidas por las pajillas de 2,5, 4,0 y 1,8 $\mathrm{ml}(51,1 \pm 2,8,48,3 \pm 3,4 \mathrm{y}$ 
Cuadro 4. Movilidad y tiempo de activación espermática postdescongelación de semen de yamú (Brycon amazonicus) crioconservado en pajillas de $0,5,1,8,2,5$ o 4,0 $\mathrm{ml}$ y descongelado a diferentes temperaturas. Los datos corresponden a la media \pm EE.

Motility and spermatic activation time post-thawing of yamú (Brycon amazonicus) sperm cryopreserved in $0.5,1.8,2.5$ or $4.0 \mathrm{ml}$ straws and thawed at different temperatures. The values correspond to the mean $\pm \mathrm{SE}$.

\begin{tabular}{|c|c|c|c|c|c|c|}
\hline \multirow{3}{*}{$\begin{array}{l}\text { Volumen } \\
\text { de la pajilla } \\
\quad(\mathrm{ml})\end{array}$} & \multicolumn{3}{|c|}{$\begin{array}{l}\text { Movilidad (\%) } \\
\quad(\mathrm{n}=13)\end{array}$} & \multicolumn{3}{|c|}{$\begin{array}{l}\text { Tiempo de activación (seg) } \\
\qquad(\mathrm{n}=7)\end{array}$} \\
\hline & \multicolumn{3}{|c|}{$\begin{array}{l}\text { Temperatura de descongelación } \\
\qquad\left({ }^{\circ} \mathrm{C}\right)\end{array}$} & \multicolumn{3}{|c|}{$\begin{array}{l}\text { Temperatura de descongelación } \\
\qquad\left({ }^{\circ} \mathrm{C}\right)\end{array}$} \\
\hline & 35 & 60 & 80 & 35 & 60 & 80 \\
\hline 0,5 & $35,3 \pm 2,3^{b}$ & $13,7 \pm 4,6^{c}$ & $7,2 \pm 2,2^{\mathrm{c}}$ & $57 \pm 4,8^{\mathrm{ab}}$ & $47,8 \pm 1,7^{\mathrm{bc}}$ & $38,6 \pm 6,1^{\mathrm{c}}$ \\
\hline 1,8 & $42,0 \pm 2,4^{\mathrm{ab}}$ & $38,9 \pm 3,1^{b}$ & $36,8 \pm 2,8^{b}$ & $59,4 \pm 5,2^{\mathrm{ab}}$ & $60,4 \pm 2,7^{\mathrm{a}}$ & $55 \pm 1,5^{\mathrm{ab}}$ \\
\hline 2,5 & $37,5 \pm 1,5^{\mathrm{b}}$ & $35,6 \pm 3,3^{b}$ & $40,0 \pm 1,0^{\mathrm{b}}$ & $59,7 \pm 7,4^{\mathrm{ab}}$ & $59,2 \pm 5,0^{\mathrm{ab}}$ & $57 \pm 3,5^{\mathrm{ab}}$ \\
\hline 4,0 & $47,0 \pm 1,6^{\mathrm{a}}$ & $40,2 \pm 2,8^{b}$ & $37,9 \pm 2,3^{b}$ & $60,2 \pm 2,4^{\mathrm{ab}}$ & $59,8 \pm 4,3^{\mathrm{ab}}$ & $55,8 \pm 3,2^{\mathrm{ab}}$ \\
\hline
\end{tabular}

a,b,c Dentro de cada variable, medias con sobreescritos distintos son significativamente diferentes $(\mathrm{P}<0,05)$.

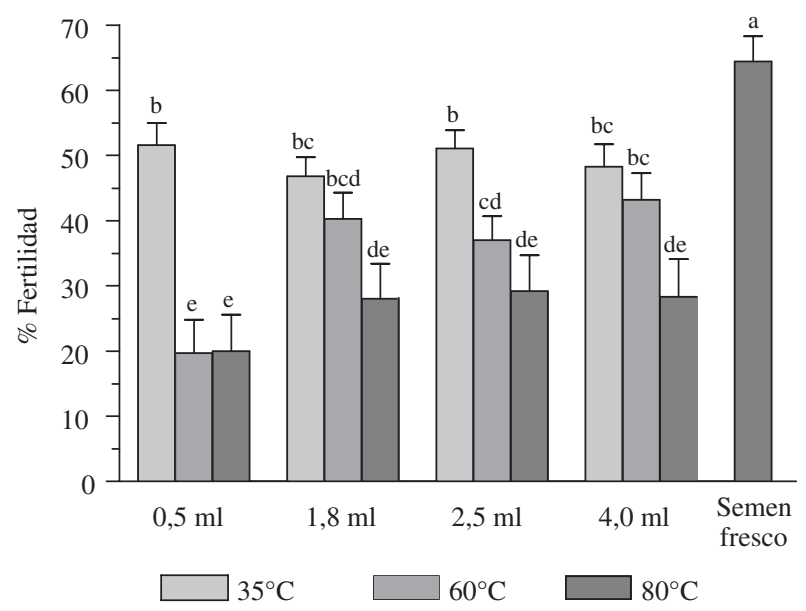

Figura 3. Fertilidad de semen de yamú (Brycon amazonicus) crioconservado en pajillas de $0,5,1,8,2,5$ o $4,0 \mathrm{ml}$ y descongelado a diferentes temperaturas $\left(35,60 \mathrm{u} 80^{\circ} \mathrm{C}\right)$. Los datos corresponden a la media $\pm \mathrm{EE}(\mathrm{n}=15)$. Barras con sobreescritos distintos difieren significativamente $(\mathrm{P}<0,05)$.

Fertility of yamú (Brycon amazonicus) sperm cryopreserved in $0.5,1.8,2.5$ or $4.0 \mathrm{ml}$ straws and thawed at different temperatures $\left(35,60\right.$ or $\left.80^{\circ} \mathrm{C}\right)$. The values correspond to the mean \pm $\mathrm{SE}(\mathrm{n}=15)$. Bars with different superscripts are significantly different $(\mathrm{P}<0.05)$.

$46,8 \pm 2,9 \%$, respectivamente) descongeladas a igual temperatura, sin observarse diferencias significativas entre éstas (figura 3). Todos los volúmenes de empaque descongelados a $80^{\circ} \mathrm{C}$ mostraron porcentajes de fertilidad inferiores $(\mathrm{P}<0,05)$ a los observados con semen descongelado a $35^{\circ} \mathrm{C}$. Por su parte, sólo las pajillas de 0,5 y $2,5 \mathrm{ml}$ descongeladas a $60^{\circ} \mathrm{C}$ mostraron fertilidad inferior $(\mathrm{P}<0,05)$ que las pajillas descongeladas a $35^{\circ} \mathrm{C}$ (figura 3 ).

\section{DISCUSION}

Para la crioconservación de semen de peces se han utilizado exitosamente los tradicionales envases o pajillas de 0,25 o 0,5 ml (Lahnsteiner y col 1995, Cruz-Casallas y col 2004); sin embargo, dentro de los procesos de fertilización artificial a escala comercial su uso se ha dificultado, debido principalmente a los grandes volúmenes de oocitos que desovan las especies tropicales cultivadas. Los sistemas de empaque de gran volumen o macrotubos ofrecen ventajas en el manejo del semen para la fertilización de los gametos, facilitando de esta forma los procesos de seminación artificial. Reportes sobre el uso de macropajillas de volúmenes mayores de $4 \mathrm{ml}$ para la crioconservación de semen de peces son limitados; Wheeler y Thorgaard (1991) utilizaron pajillas de 4,5 ml para la crioconservación de semen de trucha arco iris bajo un protocolo de 5,4\% glucosa, $10 \%$ de yema de huevo y $10 \%$ de DMSO, obteniendo porcentajes de fertilidad de $84 \%$, superiores al observado en el presente estudio. En esta misma especie, Cabrita y col (2001) observaron con pajillas de 5,0 ml tasas de fertilidad de $73 \%$ con relación al control. En Pleuronectes ferrugineus la crioconservación de semen en pajillas de 1,7 ml, utilizando $10 \%$ de propilenglicol, mostró porcentajes de fertilidad de $56,1 \%$ (Richardson y col 1999), similar a lo obtenido en este estudio con pajillas de 2,5 y 4,0 ml descongeladas a $35^{\circ} \mathrm{C}$.

La tasa de congelación para las pajillas de $0,5 \mathrm{ml}$ en los rangos establecidos fue superior a la mostrada por los macrotubos de 1,8, 2,5 y 4,0 ml, facilitando tal vez que la deshidratación celular durante la congelación haya sido menor en las pajillas de $0,5 \mathrm{ml}$ que en los macrotubos, permitiendo una mayor formación intracelular de cristales de hielo en los espermatozoides crioconservados en este tipo de envase. Por otro lado, las menores tasas de congelación de los macrotubos, con respecto a las pajillas 
de $0,5 \mathrm{ml}$, pudieron haber expuesto las células contenidas en ellas al efecto solución; sin embargo, Cabrita y col (2001) reportaron efecto solución en espermatozoides de trucha arco iris sólo cuando éstos fueron sometidos a una tasa de congelación de $8^{\circ} \mathrm{C} \min ^{-1}$ en el rango de 4 a $-20^{\circ} \mathrm{C}$, la cual es mucho más baja que la observada en el presente estudio.

Viveiros y col (2001) reportaron en semen de catfish africano (Clarias gariepinus), que cuando las tasas de congelación son muy lentas los espermatozoides son afectados por la exposición prolongada a la alta concentración de solutos y por fenómenos asociados con deshidratación excesiva, ocasionando estrés mecánico de las células. En el presente trabajo, aunque la exposición de las pajillas a los vapores de nitrógeno fue de $30 \mathrm{~min}$, la temperatura de $-100^{\circ} \mathrm{C}$ fue alcanzada relativamente rápido en cada uno de los volúmenes de empaque estudiados, disminuyendo así la probabilidad de criodaño, ya que a esta temperatura de congelación básicamente la célula espermática ha quedado metabólicamente inactiva (Mazur 1980, Holt 2000).

Todos los volúmenes de empaque, a excepción de la pajilla de $0,5 \mathrm{ml}$, presentaron un aumento transitorio de temperatura durante el proceso de congelación. Este cambio toma lugar intracelularmente cuando el agua en fase líquida pasa a sólida en forma de cristales y origina la aparición espontánea del primer núcleo de hielo (nucleación), lo cual ocurre generalmente cuando la solución llega a temperaturas entre -5 y $-15^{\circ} \mathrm{C}$ (Zachariassen y Kristiansen 2000, Viveiros y col 2001). Este núcleo, de forma hexagonal, adhiere moléculas de agua por medio de interacciones electrostáticas, promoviendo la formación de núcleos de mayor tamaño. A medida que avanza la formación de cristales de hielo se libera energía en forma de calor (calor latente) (Viveiros y col 2001), el cual produce un pico transitorio de ascenso de temperatura que, cuando es prolongado, puede ser letal para la célula.

Richardson y col (1999) determinaron que los cambios en estructura y viabilidad de los espermatozoides durante el proceso de congelación son principalmente influenciados por la longitud del pico de calor latente, ocasionado por la liberación del calor de fusión durante el cambio de temperatura de -5 a $-45^{\circ} \mathrm{C}$. Estos mismos autores observaron en Pleuronectes ferrugineus que el tiempo empleado para que la solución retornara a su temperatura original, después de la liberación del calor de fusión, es superior en pajillas de mayor volumen, pudiendo ocurrir una situación similar en el presente estudio a medida que se aumentó el volumen de empaque $(1,8,2,5,4,0 \mathrm{ml})$, especialmente en las pajillas descongeladas a $80^{\circ} \mathrm{C}$, ocasionando de esta forma disminución en la movilidad espermática; sin embargo, este efecto no se reflejó en los porcentajes de fertilidad.

Las tasas de descongelación mostradas por todos los volúmenes de empaque en las tres temperaturas de des- congelación fueron superiores a la tasa de congelación a la cual fue sometida cada pajilla. En el caso de la pajilla de $0,5 \mathrm{ml}$, las altas tasas de descongelación, especialmente a $60^{\circ} \mathrm{C}$ y $80^{\circ} \mathrm{C}$, pudieron afectar la célula espermática, reflejándose en una menor movilidad. Muldrew y McGann (1990) proponen que la ruptura de la membrana plasmática sería causada por la fricción del agua al pasar rápidamente a través de ella, excediendo su capacidad de conducirla, especialmente cuando se somete a tasas de descongelación más rápidas que de congelación.

Las condiciones de descongelación utilizadas en este estudio permitieron un proceso de descongelación completo, pudiéndose observar en todos los tratamientos movilidad espermática posdescongelación, lo cual difiere de lo reportado en trucha arco iris, cuyo semen empacado en pajillas de $5 \mathrm{ml}$ y descongelado a $25^{\circ} \mathrm{C}$ por 30 seg permaneció congelado en la fracción interna de la pajilla sin mostrar movilidad (Cabrita y col 2001).

El hecho de que las pajillas de $0,5 \mathrm{ml}$ descongeladas a $60^{\circ} \mathrm{Cu} 80^{\circ} \mathrm{C}$ mostraran movilidades espermáticas y porcentajes de fertilidad significativamente inferiores a las observadas por los otros volúmenes de empaque, podría ser también explicado por características propias del mismo sistema de empaque y por variaciones individuales de los contenidos lipídicos en la membrana plasmática de espermatozoides descongelados a altas temperaturas. Maldjiana y col (2005) observaron en espermatozoides de toro una pérdida de colesterol después de la descongelación, lo cual estuvo relacionado con inducción de la capacitación espermática y cambios en la calidad de la movilidad, afectando directamente la fertilidad. Hay que considerar que aun cuando en la evaluación de la movilidad algunos espermatozoides pueden no presentar movilidad vigorosa y progresiva, éstos pueden mantener su capacidad fecundante y ser finalmente estimulados por factores capacitantes, presentes en el fluido folicular durante el proceso de fertilización (Flesch y Gadella 2000); sin embargo, en el presente estudio se utilizó una dosis de semen crioconservado mayor que de semen fresco, las cuales fueron basadas en resultados previos obtenidos en nuestro laboratorio (Velasco-Santamaría y col 2004) y que explican también la existencia de daños subletales de la célula espermática sometida al proceso de crioconservación que no afectan la movilidad, pero que disminuyen su capacidad fecundante (Cabrita y col 1998).

En general, la disminución en la fertilidad presentada por todos los sistemas de empaque al aumentar la temperatura de descongelación pudo estar relacionada con dos aspectos importantes. Primero, aparentemente la tasa de descongelación alcanzada en el baño de agua a $35^{\circ} \mathrm{C}$ es más equilibrada en los aspectos biofísicos intracelulares, con la tasa de congelación a que fueron sometidos todos los volúmenes de empaque. Segundo, durante la congelación se presenta difusión lateral de proteínas de membra- 
na y formación de clusters en el protoplasma espermático con posterior reacomodación durante el proceso de descongelación, como lo reportado en trucha arco iris y trucha café (Drokin y col 1998) y es probable que esta reorganización sea inversamente proporcional a la tasa de descongelación utilizada. En mamíferos, esta reorganización lateral de los lípidos y proteínas en la zona ecuatorial de la cabeza del espermatozoide es necesaria para el proceso de fertilización, específicamente durante la fusión de la membrana plasmática del espermatozoide y el oocito en el espacio perivitelino previo a la fertilización (Flesch y Gadella 2000).

\section{CONCLUSION}

Los resultados indican que aunque la pajilla tradicional de $0,5 \mathrm{ml}$ presentó una tasa de congelación y descongelación diferente a la presentada por los macrotubos, los porcentajes de fertilidad alcanzados cuando se descongelaron a $35^{\circ} \mathrm{C}$ fueron similares a los observados con los otros volúmenes de empaque; sin embargo, el bajo volumen de semen que este sistema permite empacar no es práctico para los procesos de fertilización artificial de esta especie. Por otro lado, la descongelación a $60^{\circ} \mathrm{C}$ y especialmente a $80^{\circ} \mathrm{C}$ mostró una disminución significativa en las tasas de fertilidad. Por lo tanto, para propósitos de seminación artificial en yamú y en virtud de su alta fecundidad, el macrotubo de 4,0 ml descongelado a $35^{\circ} \mathrm{C}$ durante $90 \mathrm{seg}$ presenta las mejores ventajas en cuanto a su manejo en el proceso de congelacióndescongelación, calidad espermática postdescongelación y porcentaje de fertilidad, lo cual optimizaría las prácticas de seminación artificial a gran escala en esta especie.

\section{RESUMEN}

El objetivo fue establecer las condiciones de congelacióndescongelación de semen de yamú (Brycon amazonicus) empacado en pajillas de diferentes volúmenes y su efecto sobre la movilidad espermática y fertilidad postdescongelación. La inducción a la maduración final de las gónadas fue realizada con extracto de hipófisis de carpa. El semen obtenido fue evaluado y diluido (1:4) en una solución de $5,5 \%$ glucosa, $12 \%$ yema de huevo y $10 \%$ dimetil sulfóxido, empacado en pajillas de 0,5, 1,8, 2,5 o 4,0 ml y congelado en vapores de nitrógeno. La descongelación fue realizada en baño de agua a $35^{\circ} \mathrm{C}, 60^{\circ} \mathrm{C} \mathrm{u} 80^{\circ} \mathrm{C}$ durante diferentes tiempos. Todas las pajillas mostraron una tasa total de congelación similar (entre 7,5 a $\left.12,9^{\circ} \mathrm{C} \mathrm{min}^{-1}\right)$. A medida que se utilizó una mayor temperatura, la tasa total de descongelación aumentó. Las pajillas de 4,0 ml descongeladas a $35^{\circ} \mathrm{C}$ tuvieron la mayor movilidad y tiempo de activación posdescongelación (47,0 $\pm 1,6 \%$ y $60,2 \pm 2,4 \mathrm{seg})$. La fertilidad obtenida con semen congelado en todos los volúmenes de empaque fue significativamente menor $(\mathrm{P}<0,05)$ cuando comparada con semen fresco $(65 \pm 4 \%)$. La mayor fertilidad se observó con pajillas de $0,5 \mathrm{ml}$, descongeladas a $35^{\circ} \mathrm{C}(52 \pm 3 \%)$, seguida por las pajillas de $2,5,4,0$ y $1,8 \mathrm{ml}(51 \pm 3,48 \pm 3$ y $47 \pm 3 \%$, respectivamente $)$ descongeladas a igual temperatura. En conclusión, pajillas de 4,0 ml, descongeladas a $35^{\circ} \mathrm{C}$, mostraron las mejores características postdescongelación y tasas de congelación-descongelación más homogéneas, comparado con los otros empaques. Por su parte, la fertilidad no fue afectada por el volumen de empaque cuando se utilizaron $35^{\circ} \mathrm{C}$ como temperatura de descongelación.

\section{AGRADECIMIENTOS}

Los autores agradecen a COLCIENCIAS-SENA proyecto código 1122-09-12396, contrato RC. No 397-2002 Universidad de los LlanosCOLCIENCIAS por el apoyo financiero y al Instituto de Acuicultura de los Llanos por el apoyo logístico.

\section{REFERENCIAS}

Billard R. 1998. Artificial insemination and gamete management in fish. Mar Behave Physiol 14, 3-21.

Cabrita E, R Alvarez, L Anel, K J Rana, M P Herráez. 1998. Sublethal damage during cryopreservation of rainbow trout sperm. Cryobiology 37, 245-253.

Cabrita E, V Robles, R Alvarez, M P Herráez. 2001. Cryopreservation of rainbow trout sperm in large volume straws: application to large scale fertilization. Aquaculture 201, 301-314.

Cruz-Casallas P E, S P Pardo-Carrasco, J A Arias-Castellanos, P E Lombo-Castellanos, D A Lombo-Rodríguez, J E Pardo-Mariño. 2004. Cryopreservation of Yamú Brycon siebenthalae Milt. J World Aquaculture Soc 35, 529-535.

Cruz-Casallas P E, Y M Velasco-Santamaría, V M Medina-Robles. 2006. Cryopreservation of yamú (Brycon amazonicus) sperm for large scale fertilization. Aquaculture 256, 264-271.

Drokin S, H Stein, H Bartscherer. 1998. Effect of cryopreservation on the fine structure of spermatozoa of Rainbow Trout (Oncorhynchus mykiss) and Brown Trout (Salmo trutta F. Fario). Cryobiology 37, 263-270.

Flesch F M, B M Gadella. 2000. Dynamics of the mammalian sperm plasma membrane in the process of fertilization. Biochim Biophys Acta 469, 197-235.

Holt W V. 2000. Basic aspects of frozen storage of semen. Anim Reprod Sci 62, 3-22.

Lahnsteiner F, T Weismann, R A Patzner.1995. Uniform method for cryopreservation of semen of the salmonid fishes (Oncorhynchus mykiss, Salmo trutta fario, Salmo trutta lacustris, Coregonus sp). Aquaculture Res 26, 801-807.

Lahnsteiner F, T Weismann, R A Patzner. 1996. Cryopreservation of semen of the grayling (Thymallus thymallus) and the Danube salmon (Hucho hucho). Aquaculture 144, 265-274.

Lahnsteiner F, T Weismann, R A Patzner. 1997. Methanol as cryoprotectant and the suitability of $1.2 \mathrm{ml}$ and $5.0 \mathrm{ml}$ straws for cryopreservation of semen from salmonid fishes. Aquaculture Res 28, 471-479.

Lahnsteiner F. 2000. Semen cryopreservation in the Salmonidae and in the Northern pike. Aquaculture Research 31, 245-258.

Maldjiana A, F Pizzic, T Gliozzic, S Cerolinib, P Pennya, R Noblea. 2005. Changes in sperm quality and lipid composition during cryopreservation of boar semen. Theriogenology 63, 411-421.

Mazur P. 1980. Fundamental aspects of the freezing of cells, whit emphasis on mammalian ova and embryos. Proceedings of the $9^{\text {th }}$ International Congress on Animal Reproduction and Artificial Insemination, Madrid, España, Pp 99-114.

Medina-Robles V M, Y M Velasco-Santamaría, P E Cruz-Casallas. 2005. Aspectos generales de la crioconservación espermática en peces teleósteos. Rev Col Cienc Pec 18, 34-48.

Muldrew K, L E McGann. 1990. Mechanisms of intracellular ice formation. Biophys J 57, 525-532.

Ogier de Baulny B, Y Le Verne, D Kerboeuf, G Maisse. 1997. Flow cytometric evaluation of mitochondrial activity and membrane integrity in fresh and cryopreserved Rainbow trout (Oncorhynchus mykiss) spermatozoa. Cryobiology 34, 141-149. 
Richardson G F, C E Wilson, L W Crim, X Z Yao. 1999. Cryopreservation of yellowtail flounder (Pleuronectes ferrugineus) semen in large straws. Aquaculture 174, 89-94.

Velasco-Santamaría Y M, P E Cruz-Casallas, A H Calderón-Fonseca. 2004. Inseminación artificial en yamú (Brycon siebenthalae): determinación de la dosis inseminante con semen fresco y crioconservado. Memorias II Congreso Colombiano de Acuicultura, $\mathrm{X}$ Jornada de Acuicultura. Instituto de Acuicultura de la Universidad de los Llanos, Villavicencio, Meta, Colombia, Pp 105-107.
Viveiros A T, E J Lock, H Woelders, J Komen. 2001. Influence of cooling rates and plunging temperatures in an interrupted slowfreezing procedure for semen of the African catfish, Clarias gariepinus. Cryobiology 43, 276-287.

Wheeler P, G Thorgaard. 1991. Cryopreservation of rainbow trout semen in large straws. Aquaculture 93, 95-100.

Zachariassen K E, E Kristiansen. 2000. Ice nucleation and antinucleation in nature. Cryobiology 41, 257-279. 
